\title{
Filigrane
}

Écoutes psychothérapiques

\section{Réflexions d'une psychanalyste sur l'enfant dans la société occidentale d'aujourd'hui}

\section{Florence Guignard}

Volume 19, numéro 1, printemps 2010

Adieu Oedipe, bonjour Narcisse ?

URI : https://id.erudit.org/iderudit/044176ar

DOI : https://doi.org/10.7202/044176ar

Aller au sommaire du numéro

Éditeur(s)

Revue Santé mentale au Québec

ISSN

1192-1412 (imprimé)

1911-4656 (numérique)

Découvrir la revue

Citer cet article

Guignard, F. (2010). Réflexions d'une psychanalyste sur l'enfant dans la société occidentale d'aujourd'hui. Filigrane, 19(1), 11-27.

https://doi.org/10.7202/044176ar

\section{Résumé de l'article}

Psychanalyste d'enfants, d'adolescents et d'adultes, l'auteur examine les conséquences des modifications structurelles que connaît la société occidentale d'aujourd'hui, où le fantastique développement des techniques de communication vient s'ajouter à la fragilisation des structures familiales. Autant de facteurs nouveaux auxquels doit se confronter le développement des enfants d'aujourd'hui. Elle constate que le tissage entre les différents degrés de relation sociale et la relation de véritable intimité s'est délité et désymbolisé. Cet état incertain des relations humaines confronte l'individu à des exigences nouvelles dans l'économie de ses pulsions et, partant, à de nouvelles formes d'angoisse. La mondialisation de la communication virtuelle modifie radicalement le rapport à la réalité et à la vérité et augmente le risque de voir la « mentalité de groupe " prendre le dessus sur la pensée personnelle. Elle se centre sur les conséquences de la quasi-disparition, chez les enfants d'aujourd'hui en Occident, de la " période de latence » dont Freud, voici un siècle, faisait le socle de sa théorie du développement en deux temps de la sexualité humaine, du refoulement, de l'après-coup et, par conséquent, de sa théorie de la névrose infantile et de la névrose de transfert. Sur ce sujet d'une haute importance tant clinique que théorique, elle développe des pistes de réflexion d'ordre phénoménologique, dynamique, économique et structural, sans oublier les conséquences de cet état de choses sur le champ analytique transféro-contretransférentiel.
Ce document est protégé par la loi sur le droit d'auteur. L'utilisation des services d'Érudit (y compris la reproduction) est assujettie à sa politique d'utilisation que vous pouvez consulter en ligne.

https://apropos.erudit.org/fr/usagers/politique-dutilisation/ 


\title{
Réflexions d'une psychanalyste sur I'enfant dans la société occidentale d'aujourd'hui
}

\author{
Florence Guignard
}

\begin{abstract}
Psychanalyste d'enfants, d'adolescents et d'adultes, l'auteur examine les conséquences des modifications structurelles que connaît la société occidentale d'aujourd'hui, où le fantastique développement des techniques de communication vient s'ajouter à la fragilisation des structures familiales. Autant de facteurs nouveaux auxquels doit se confronter le développement des enfants d'aujourd'hui. Elle constate que le tissage entre les différents degrés de relation sociale et la relation de véritable intimité s'est délité et désymbolisé. Cet état incertain des relations humaines confronte l'individu à des exigences nouvelles dans l'économie de ses pulsions et, partant, à de nouvelles formes d'angoisse. La mondialisation de la communication virtuelle modifie radicalement le rapport à la réalité et à la vérité et augmente le risque de voir la "mentalité de groupe " prendre le dessus sur la pensée personnelle. Elle se centre sur les conséquences de la quasi-disparition, chez les enfants d'aujourd'hui en Occident, de la " période de latence " dont Freud, voici un siècle, faisait le socle de sa théorie du développement en deux temps de la sexualité humaine, du refoulement, de l'aprèscoup et, par conséquent, de sa théorie de la névrose infantile et de la névrose de transfert. Sur ce sujet d'une haute importance tant clinique que théorique, elle développe des pistes de réflexion d'ordre phénoménologique, dynamique, économique et structural, sans oublier les conséquences de cet état de choses sur le champ analytique transféro-contretransférentiel.
\end{abstract}

J'estime que l'on ne doit pas faire de théories - elles doivent tomber à l'improviste dans votre maison, comme des hôtes qu'on n'avait pas invités, alors qu'on est occupé à l'examen des détails...

Freud, Lettre à Ferenczi du 31 juillet 1915, soit trois jours après l'envoi à celui-ci du manuscrit de «Vue

d'ensemble des névroses de transfert » (Freud,

1915).

\section{Introduction}

a société occidentale connaît des modifications structurelles dont le rythme va croissant. Le fantastique développement des techniques de communication et de 
leurs applications vient s'ajouter à la fragilisation et aux profonds changements des structures familiales pour constituer autant de facteurs nouveaux auxquels doit se confronter le développement des enfants d'aujourd'hui.

Cet essai fait état de mes nombreuses interrogations. Il pose infiniment plus de questions qu'il n'en résout; il ne constitue en aucun cas un point formel et catégorique sur l'état d'un problème dont je ne suis pas en mesure d'évaluer tous les paramètres présents et, surtout, à venir. Je pense qu'il faudra beaucoup de temps et de travail en commun pour approfondir les questions que je soulève ici.

Ma réflexion actuelle porte sur le double impact, social et numérique, qui touche tous les enfants de la fin du vingtième siècle et, plus encore, ceux de ce début de troisième millénaire, entraîne des changements considérables dans les processus de développement et les modes d'adaptation proposés à leur fonctionnement psychique par leur environnement. Il s'agit d'un phénomène de deuxième, voire de troisième génération, qui a des effets spécifiques et durables auxquels le psychanalyste est confronté toutes les fois qu'il est sollicité pour examiner ou soigner un enfant.

\section{L'avènement de la personne humaine dans la société dont il fait partie}

Les relations de l'individu avec la société sont fonction de l'évolution respective de chacune des deux parties. Or, la société occidentale d'aujourd'hui n'a plus grandchose à voir avec la société d'Europe centrale dans laquelle Freud s'est développé. Dans le système de valeurs occidental actuel, le développement de la vie psychique de l'individu - champ dont s'occupe la psychanalyse - est devenu d'une importance très secondaire par rapport aux critères d'efficacité et d'adaptation à un environnement qui, pour sa part, s'est modifié à grande vitesse au cours des dernières décades. Le fantastique développement des moyens d'information - et de désinformation - immédiate de ce qui se passe dans le monde entier s'allie à l'accroissement tout aussi considérable des moyens de communication avec «d'autres", dont il est souvent difficile, voire impossible, de savoir s'il s'agit d'êtres humains réels, de leurs « avatars » ou de robots plus ou moins perfectionnés ${ }^{1}$. Le tissage subtil qui existait traditionnellement entre les différents degrés de relation sociale et la relation de véritable intimité, à soimême et à ses plus proches, s'est délité et, par là même, désymbolisé. Cet état incertain des relations humaines confronte l'individu à des exigences nouvelles dans l'économie de ses pulsions et, partant, à de nouvelles formes d'angoisse (Castoriadis, 1996). Dans ce contexte, la mondialisation de la communication virtuelle ne peut se produire sans modifier radicalement le rapport à la vérité, ni augmenter le risque de voir la «mentalité de groupe»(Bion, 1959), prendre le dessus sur la pensée personnelle.

Il faudra plusieurs générations pour parvenir à intégrer cette néo-réalité qu'est le virtuel dans une civilisation qui, durant cette période, sera influencée par elle dans des proportions et des directions qu'il est impossible d'évaluer à court terme. La question de la transmission intergénérationnelle se pose donc dans des termes nouveaux. Issu de parents nés durant les «trente glorieuses ${ }^{2}$ » un jeune intellectuel n'a-t-il pas déclaré récemment vouloir écrire un livre qu'il intitulerait: Parents, vous ne nous avez rien transmis... ? Que penser de cet état de choses? 


\section{Le virtuel et les principes du fonctionnement psychique}

L'accélération des technologies d'information, de communication et de simulation ne modifie pas seulement nos actes, mais également notre manière de penser. Le monde de l'Internet permet d'explorer la quasi totalité du savoir humain et de diffuser presque instantanément les découvertes effectuées dans les domaines les plus divers et les plus spécialisés. Les développements de l'intelligence artificielle et des nanotechnologies, les recherches en biologie, en génétique et en psychopharmacologie posent en particulier des questions fondamentales sur l'identité de l'être humain, qui se situe aujourd'hui dans une relation nouvelle avec une société humaine ayant elle-même considérablement changé.

Du point de vue de la communication, la civilisation de l'Internet favorise des échanges directs d'un être humain avec d'autres, hors de son milieu de vie habituel et ce, en dépit des distances tant géographiques que sociologiques. Il modifie profondément et de façon très nouvelle la position de l'individu face au sentiment de solitude, sentiment dont l'élaboration fut considérée jusqu'ici comme inhérente au développement psychique normal. Du même coup, l'investissement qu'il fait de sa vie psychique interne et de son autonomie de pensée se modifie selon des paramètres nouveaux dont nous ne possédons pas encore la véritable mesure et dont nous ne pouvons pas encore estimer la véritable portée pour le devenir de la structure psychique humaine.

Ainsi, le psychanalyste se doit de confronter le monde du virtuel à ses critères métapsychologiques de base, dans l'espoir de comprendre le premier en étendant le champ des seconds. Plusieurs d'entre nous s'y attachent depuis quelques années déjà ${ }^{3}$ (Turkle, 1997; Tisseron, 2006; Tisseron et al., 2007).

Paraphrasant la fameuse question de l'architecte Louis Kahn: «que veut la brique?», Sherry Turkle se demande : «que veut la simulation?» Sa réponse est éloquente: «Les simulations demandent, voire exigent une immersion, et le bénéfice en est clair. Les architectes construisent des édifices qui étaient inimaginables avant le dessin virtuel ; les scientifiques déterminent la structure des molécules en les manipulant dans l'espace virtuel; les médecins pratiquent l'anatomie sur des humains numérisés. Cependant, immergés dans la simulation, nous sommes vulnérables. Il y a autant de pertes que de gains. Les scientifiques plus âgés décrivent une jeune génération «saoulée par le code». Les jeunes scientifiques, ingénieurs et dessinateurs, citoyens à part entière du monde virtuel, se démènent pour saisir la connaissance tacite des édifices et des corps que possèdent leurs mentors. Sur les deux rives d'un fossé générationnel, il existe l'angoisse qu'avec la simulation quelque chose d'important soit en train de nous échapper» (Turkle, 2009).

Pour ma part, je commencerai par énumérer quelques thèmes de réflexion, me réservant de revenir plus en détail sur certains d'entre eux dans la suite de ces réflexions:

1. Le monde du virtuel permet et favorise des échanges directs d'un individu avec d'autres qui, jusque-là, lui auraient été totalement inaccessibles, pour des raisons tant géographiques que sociologiques. Dans le jeu de ces 
rencontres virtuelles, les règles, implicites ou explicites, incitent à travestir et à escamoter le rôle de la différence des sexes et des générations. Ces deux critères ne jouent donc plus le rôle traditionnel que nous lui avons connu et dont la reconnaissance est partie prenante, pour le psychanalyste, de l'accomplissement du Complexe d'CEdipe. On peut ainsi se questionner sur le devenir de cette organisation de base de la névrose infantile.

2. Ce nouveau champ très étendu de relations qu'est le virtuel a une incidence sur le réseau des relations géographiquement et psychiquement plus proches que sont la famille et l'environnement scolaire et social de l'enfant. On peut donc se demander si, et comment, il interfere avec la structuration des capacités affectives et relationnelles de l'enfant. En effet, la communication proposée par le virtuel engage davantage l'investissement des connaissances et des performances, plutôt que celui des émotions et du développement de la pensée qui en découle (Bion, 1961).

3. Le virtuel entretient avec le principe de plaisir/déplaisir et le principe de réalité des relations fort différentes de celles qu'entretient le fantasme: alors qu'il faut une organisation projective très intense, voire pathologique, pour que le sujet confonde le fantasme avec la réalité, le virtuel propose une illusion de réel qui permet de faire l'économie du travail psychique de liens et de transformation nécessaire aux bons rapports entre le monde psychique interne et la réalité extérieure.

4. L'univers du virtuel est un univers de simulation et, en tant que tel, il rend d'immenses services à la recherche dans toutes sortes de domaines. Il lui manque cependant le critère même du développement de l'identité individuelle: «apprendre l'incertitude» (Guignard, 1990), accepter l'aléatoire. Tous les tenants des jeux vidéo vantent les qualités de maîtrise et la revalorisation narcissique rendue possible par leur pratique. La dimension relationnelle y est implicitement traitée comme une valence à gérer, davantage que comme une composante du développement psychique de la personnalité et de la qualité de la relation d'objet. C'est un changement de vecteur non négligeable qui reflète bien le changement de société que nous sommes en train de vivre. Seule la théorie quantique (Ortoli et Pharabod, 1984) échappe à cette mentalité de gestionnaire qui vient se poser de plus en plus comme un critère majeur dans la nouvelle éducation des enfants et des adolescents d'aujourd'hui.

5. Dans le domaine de la représentation, le virtuel oblige le psychanalyste à effectuer un changement de perspective. En effet, sur l'axe perception représentation, la civilisation du virtuel place le curseur beaucoup plus près de la perception que de la représentation, en imposant à la perception du spectateur une quantité infiniment plus importante d'icônes (Peirce) dont les relations avec le monde réel sont plus ou moins subtilement décalées.

6. Mais, simultanément, la perception est sollicitée à s'exercer sur un monde pseudo réel, ce qui propose une ligne de fuite non négligeable lorsque la 
réalité qui se propose à la perception est trop douloureuse. La passivité de l'enfant est tout naturellement sollicitée. On peut donc parler ici de «clivage auto narcissique » (Ferenczi, 1932) ou de " clivage passif» (Meltzer, 1977) et s'intéresser au contenu et au devenir de chacune des deux parties clivées.

7. La partie clivée visible est sollicitée à l'action par les jeux vidéos, ainsi qu'à une forme bien spécifique de pensée, qui est la stratégie. Or, un bon stratège sait faire taire ses sentiments. Le développement de la pensée à partir des émotions en prend un sale coup, ainsi que les processus de défense secondaires (déplacement, négation et refoulement notamment).

8. Il est beaucoup plus difficile de se faire une représentation de la partie clivée invisible, sinon que les auteurs cités plus haut y voient à la fois le lieu du véritable self du sujet, et le lieu par excellence des mécanismes de défense primaire (clivage, déni, idéalisation et projection identificatoire).

9. Cette prévalence du clivage passif sur les mécanismes de défense secondaires de l'ordre du refoulement joue un rôle important dans la déstructuration, voire la possible disparition de la structure névrotique.

10. Par ailleurs, le développement du «monde de l'image imposée à la perception visuelle» bouleverse la place et le rôle du langage dans la représentation du monde. Le monde proposé à la perception étant un monde d'icônes prédigérées, on pourrait dire que, du point de vue du monde psychique interne, ces images imposées sont des pseudo représentations, dans lesquelles les objets proposés sont des pseudo objets, dont les liens avec les objets internes du sujet demeurent inconnues et, à la limite, sans importance. La tentation est grande, pour le sujet, de substituer le «monde de l'image imposé à la perception visuelle» au «monde psychique interne de la représentation en lien émotionnel avec ses objets internes ». Du coup, le langage intérieur n'a plus la nécessité de se développer et son rôle dans l'exploration des relations du sujet avec ses objets internes diminue d'autant.

11. Je ne pense pas que l'on puisse considérer l'espace virtuel comme un espace transitionnel, comme cela a été souvent proposé. Je ne crois pas que Winnicott eût été d'accord avec cette extension de son concept. La différence majeure réside dans la question de la relation d'objet : un objet transitionnel a les qualités mélangées de la mère et du sujet bébé, à partir de quoi chacun des deux protagonistes élabore la perte de la symbiose primaire (Bleger, 1981) et (re) construit son identité. Un objet virtuel a les qualités que le programmateur a voulu lui donner - avec, certes, une part de son inconscient - et si l'enfant entre en relation avec ces qualités, cela ne le dispensera pas pour autant d'avoir à élaborer sa relation avec sa mère réelle.

\section{Le psychanalyste face aux changements de société}

Depuis une vingtaine d'années, on observe un net changement dans la population qui, dans les métropoles occidentales, s'adresse à des psychanalystes dans l'espoir 
de soulager une souffrance psychique. Tout se passe comme si le modèle classique de la névrose, établi par Freud, concernait de moins en moins de patients, tout spécialement dans les plus jeunes générations.

Freud a toujours lié ses découvertes du fonctionnement psychique au tissu social dans lequel il a observé et soigné ses patients. Il ne serait donc nullement étonné de constater avec nous combien la psychopathologie individuelle d'aujourd'hui s'est modifiée, en même temps que se délitent les structures sociales et familiales de notre cité occidentale actuelle.

Qu'il traite des adultes, des adolescents ou des enfants, on sait que le souci du psychanalyste concerne le développement du Moi et l'amélioration de la nature des défenses qui interviennent dans les relations du sujet avec ses propres pulsions et celles des autres. Dans cette perspective, le travail analytique se centre essentiellement sur la compréhension, dans le champ analytique et au travers du transfert, des relations du Moi de l'analysant avec ses propres objets internes, projetés sur l'analyste, nous souvenant que les objets internes ont tous un rôle dans la constitution et la qualification du Surmoi.

Il est donc légitime de se demander si ces buts de la psychanalyse rencontrent aujourd'hui des structures, tant individuelles que sociales, suffisamment analogues à celles qu'analysait Freud voici un siècle, pour que le corpus psychanalytique puisse, sinon demeurer en l'état, du moins nous servir encore de référence pour notre exploration du psychisme humain.

$\mathrm{Au}$ cours des soixante dernières années, les psychanalystes ont élargi leurs connaissances sur l'articulation des pulsions sexuelles avec les pulsions du Moi. L'influence de l'œuvre de Melanie Klein a permis de reconnaître l'importance du rôle de l'objet dans le fonctionnement pulsionnel, notamment celui du tout premier objet d'amour et de haine du petit d'homme: la mère. Qu'il s'agisse des concepts winnicottiens de holding et de handling (Winnicott, 1958), du concept bionien de capacité de rêverie de la mère ou de la théorisation laplanchienne (Guignard, 2006) de la séduction maternelle, il est désormais établi que les orientations et les transformations des pulsions sexuelles du nourrisson à l'intérieur du couple qu'il forme avec sa mère participent de manière essentielle à l'organisation du premier Moi de l'enfant. De l'écoute que lui fournira la mère, dans sa relation avec elle comme dans sa relation avec le père et avec la fratrie, dépendra la place de l'individu devenu adulte dans la société dont il sera partie prenante.

Dans le même temps, les travaux des post-freudiens, notamment ceux de l'École Anglaise et de l'École Argentine, ont permis d'explorer et de soigner des régions du psychisme plus proches du fonctionnement psychotique que du fonctionnement névrotique. Au fil des ans, on a constaté que les psychanalystes avaient de plus en plus affaire à une "pathologie des limites » : limites entre soi et autrui, entre penser et agir, entre la réalité psychique et la réalité extérieure, et, depuis quelques années, entre le virtuel et le réel. Fragiles, mal organisées, ces limites se désintègrent d'autant plus facilement que les limites de la société environnante se sont elles-mêmes assouplies, fragilisées, désorganisées. 
Il est grand temps de ne pas regarder seulement ces patients «états limite» comme «différents » et posant des problèmes d'indication et de technique, mais bien de les considérer comme l'occasion qui nous oblige à reconsidérer nos modèles.

Certes, le psychanalyste dispose actuellement de paramètres théorico-techniques plus fins que ceux de la première moitié du Xxe siècle. Il peut observer et analyser des mouvements psychiques plus complexes, tant dans leur nature que dans leurs intrications.

Il faut néanmoins se poser la question suivante : l'évolution de la société vat-elle, ou non, modifier les paramètres de base de la cure psychanalytique?

Toute la problématique du devenir du désir, de la culpabilité, du refoulement et des identifications, est contenue dans cette interrogation.

\section{Questionner nos concepts}

Cent ans après la parution des Trois Essais sur la théorie de la sexualité (Freud, 1905), tandis que de nouvelles attaques virulentes viennent remettre en question le bien-fondé de la technique analytique et de l'appareil théorique sur lequel il s'étaie, il est important que les psychanalystes eux-mêmes puissent prendre la mesure des principaux concepts de leur discipline et en dégager les changements, mais aussi les invariants. Cela conduit à poser les deux questions suivantes:

- La névrose infantile et la névrose de transfert font-elles toujours partie des invariants, aujourd'hui comme en 1905, lors de la parution du texte princeps dans lequel Freud découvre et décrit l'existence et le rôle de la sexualité infantile pour le fonctionnement psychique?

- Dans le cas contraire, quels sont les éléments de ces deux concepts qui pourraient demeurer des invariants pour les psychanalystes d'aujourd'hui?

Cette référence aux Trois Essais s'impose du fait que, sans les découvertes de Freud sur la sexualité infantile, les concepts même de psychanalyse et de névrose perdent tout leur sens. Mais elle entraîne, du coup, une autre question:

- Le statut et le mode de fonctionnement de la sexualité infantile dans le développement psychique humain et dans sa psychopathologie sont-ils exactement les mêmes aujourd'hui qu'en 1905?

Certains praticiens de la psychothérapie ont opté pour la solution radicale de «jeter l'enfant avec l'eau du bain» et ont envoyé promener la pensée psychanalytique pour placer leurs espoirs dans des techniques visant à modifier le comportement et la manière consciente de faire fonctionner la pensée.

D'autres, dont je suis, tiennent à poursuivre l'investigation des processus inconscients à partir de ses invariants afin d'étudier le fonctionnement psychique à travers lequel la pulsion, héritière humaine de la sexualité, trouve ses voies de frayage pour organiser la complexité d'un sujet dans le monde d'aujourd'hui.

On ne devrait pas considérer la théorie psychanalytique comme un roc inamovible, mais bien plutôt comme un ensemble de modèles, dont il importe de remettre en question et de requalifier constamment les configurations conceptuelles à l'aune des avancées de la méthode et des modifications de la technique (cf. citation 
en exergue), mais aussi à la lumière de l'observation des changements sociologiques survenus depuis la naissance de notre discipline.

Le praticien de la psychanalyse se trouve ainsi confronté à de nouvelles responsabilités et à des exigences techniques nouvelles.

\section{Enfant roi, ou objet narcissique ${ }^{4}$ ?}

C'est sur l'enfant que va se produire l'impact le plus violent du monde du virtuel, l'enfant qui, du fait de son immaturité émotionnelle et intellectuelle, connaît déjà les difficultés inhérentes à la croissance pour mettre en place une capacité de symbolisation, laquelle, rappelons-le, requiert une relation à trois termes: le Moi, le symbole et l'objet symbolisé (Segal, 1957).

Un enfant est naturellement tributaire des générations qui le précèdent et, au tout premier plan, de la génération de ses parents. Or, on assiste aujourd'hui à une importante dérive de l'Infantile (Guignard, 1996) des adultes qui, théoriquement en charge d'éduquer la génération suivante, se servent en réalité des plus jeunes pour y projeter et y satisfaire leur propre hédonisme infantile.

Tel ce père, qui parvient à la caisse d'un supermarché, accompagné d'un petit garçon de quatre ans très excité, les bras et le caddie débordants de jouets au-dessus desquels trônait une pizza pour deux personnes, et qui demande complaisamment à son fils: «Bon, ça va? Tu as bien choisi tout ce que tu voulais?».

Cette image caricaturale illustre la primauté accordée par l'adulte d'aujourd'hui à la satisfaction immédiate de ses propres désirs infantiles au travers de ses rejetons. Il y manque la dimension essentielle de toute civilisation : un espace-temps de latence entre la formation d'un désir et sa satisfaction.

De tels changements ont des causes multiples, dont je ne saurais évidemment repérer que celles qui touchent de plus près à mon expérience clinique. Je pense notamment aux modifications considérables des circonstances et des personnes qui président à l'avènement, chez l'infans de quatre à six mois, de la découverte de l'altérité de la mère et de l'existence du tiers «paternel ». En effet, l'enfant occidental d'aujourd'hui commence très tôt sa vie en société: crèche dès l'âge de trois mois, école maternelle dès trois, voire deux ans. L'imprégnation de l'enfant par un tissu familial est réduite à la portion congrue en raison, d'une part, du peu de temps passé à la maison et, d'autre part, de la transformation grandissante de la structure de la famille. Il n'existe que peu, ou plus, de famille élargie à proximité du lieu de vie de l'enfant, et les grands-parents eux-mêmes sont souvent désunis et/ou encore en activité professionnelle.

Par ailleurs, l'enfant se voit de plus en plus souvent obligé de cliver précocement ses investissements parentaux, du fait de l'évolution de la vie des couples. Ses géniteurs sont souvent unis pour une période très brève, pour se séparer ensuite et, éventuellement, réorganiser leur vie amoureuse chacun de leur côté, dans des familles dites « recomposées », homo- ou hétérosexuelles, quand la mère ne demeure pas seule 
avec son enfant, comme c'est de plus en plus souvent le cas. Le «tiers paternel», indispensable à l'enfant pour sortir de la symbiose (Bleger, 1981) et organiser une problématique œdipienne, prend donc actuellement des formes extrêmement floues et changeantes, dans lesquelles le groupe social et sa «mentalité de groupe » remplacent l'apport du couple parental d'origine.

En imposant ces changements radicaux et souvent multiples à leurs enfants, les parents n'échappent pas à un fort sentiment de culpabilité, plus ou moins conscient. Ils vont tenter de calmer cette culpabilité en accordant des compensations matérielles à leurs chers petits, espérant pallier ainsi les carences affectives qu'ils leur font subir. Les retrouvailles avec l'enfant - un week-end, une moitié de semaine, ou une semaine sur deux - ravivent pour le parent séparé le conflit avec son ex-conjoint, et ces ruptures continuelles ne favorisent guère une continuité éducative, dont on sait le rôle qu'elle joue dans la formation du Surmoi, mais aussi de l'Idéal du Moi. Plus que jamais, l'enfant est en mesure d'obtenir la satisfaction immédiate de ses désirs matériels, qu'il apprend vite à exprimer en lieu et place de son besoin d'écoute et de contenance. Il tire très vite parti de la situation de clivage qu'on lui impose, et joue sur les deux tableaux de sa famille d'origine éclatée.

Par ailleurs, il est de notoriété publique que les défaillances de l'éducation familiale placent les enseignants dans une situation paradoxale d'éducateurs sans autorité ni mandat, de sorte qu'ils sont souvent contestés par les parents, brièvement réunis dans leur revendication de géniteurs d'un enfant en difficulté scolaire ou comportementale.

\section{Latence, refoulement et après-coup}

En installant ses modèles - complexe d'CEdipe, complexe de castration, névrose infantile prototypique de la névrose de transfert - Freud s'est basé sur sa découverte d'un développement en deux temps de la sexualité infantile, deux temps séparés par une période dite de latence, au cours de laquelle ses intérêts pulsionnels se détourneraient de la recherche d'une satisfaction directe pour se tourner davantage vers l'univers des connaissances.

Repère de l'organisation psychique en devenir, résultante de la formation d'un Surmoi-Idéal au travers des identifications au couple des parents et aux représentants des générations antérieures, la double reconnaissance de la différence des sexes et des générations constituait le relais identificatoire à des parents garants de la suprématie du principe de réalité sur le principe de plaisir/déplaisir. Ainsi la puberté advenait-elle sur un terrain déjà "cultivé », dans tous les sens du terme, fournissant des limites à l'après-coup de la crise identitaire de l'adolescence.

Or, confrontée à la société occidentale d'aujourd'hui, cette description contient en elle-même sa caducité. Pour moi, qui suis confrontée quotidiennement à l'écoute des enfants, des adolescents, mais aussi des adultes qui s'en occupent - patients adultes, parents, et psychothérapeutes de l'enfance - je peux affirmer que le développement psychique des enfants dans notre société a davantage changé au cours de ces quinze dernières années qu'il ne l'a fait au cours des cinquante années qui les ont précédées. 
J'ai découvert qu'un consensus inattendu rassemble tous les praticiens de la psychanalyse avec l'enfant et l'adolescent que j'ai interrogés au cours de ces dix dernières années dans plusieurs places européennes, nord-américaines et latino-américaines: tous s'accordent à reconnaître que la période de latence telle que Freud l'a définie en 1905 dans les Trois Essais est en voie de disparition dans le tissu social occidental actuel.

À partir des années 70, les psychanalystes ont vu s'estomper, voire s'escamoter, la différence des sexes et des générations dans les liens familiaux comme dans les liens sociaux qui se sont noués entre les individus. La remise en cause salutaire d'un certain mode d'éducation a connu son effet pervers: l'inhibition et la transformation des pulsions ont cessé de représenter des valeurs reconnues et transmises, pour devenir plutôt des tares dont il faudrait se libérer.

Une fois débarrassé de la fonction contraignante/contenante de l'exigence éducative, le sadisme primaire (Guignard, 2002a) des enfants des nouvelles générations a dû se constituer d'autres barrières défensives contre la désintrication pulsionnelle. En raison de l'évolution de la cellule familiale, ces barrières sont devenues actuellement de nature groupale, plutôt que familiale, et cet état de fait pose des problèmes nouveaux à la société et à la communication des individus entre eux.

Les syndromes d'hyperactivité se multiplient et, s'ils font l'affaire de la production pharmaceutique, ils ne sont pas, pour autant, calmés sans dommages pour le développement psychique des jeunes patients (Salomonsson, 2006).

On n'observe plus de «refroidissement» de l'expression pulsionnelle chez les enfants de six à douze ans qui, plutôt qu'une déflexion de leurs pulsions sexuelles vers des activités de sublimation, manifestent une excitabilité aussi importante que les enfants de trois à cinq ans, d'âge dit « œedipien ", tout en imitant à l'envi les attitudes et les comportements sexuels des enfants pubères, des adolescents et des jeunes adultes.

Il est donc légitime de considérer que la situation d'aujourd'hui remet en question la centralité du concept de névrose en tant que modèle économique du développement psychique.

\section{Conséquences pour le traitement psychanalytique}

Est-ce à dire que la psychanalyse est devenue caduque en tant que méthode thérapeutique et d'investigation du psychisme humain? Je ne le pense nullement.

Je vais tenter d'examiner brièvement, sous trois angles principaux, les conséquences d'une telle situation du point de vue de l'approche psychanalytique de l'enfant.

1. Angle phénoménologique;

2. Angle dynamique et économique;

3. Angle topique et structural.

\section{Angle phénoménologique}

Les pulsions épistémophiliques ne s'organisent plus essentiellement autour du fantasme originaire de scène primitive qui oriente la curiosité et le désir de com- 
prendre vers la somme de connaissances détenues - en fantasme ou en réalité - par le couple des parents et, en deçà, par l'histoire de la pensée humaine. Plutôt que de se développer dans la mise en place de la capacité de symbolisation, ces pulsions, drainées par le formidable développement du monde du virtuel, s'orientent avant tout vers l'action, dont nous savons qu'elle requiert un système de logique binaire.

Or, en ouvrant directement sur la mise en acte de la solution sélectionnée, le système de logique binaire ramène le sujet au niveau primaire du principe de plaisir/déplaisir, tel que Freud l'a décrit en 1925 : «bon, à avaler, mauvais, à recracher».

Cette solution par l'action, qui court-circuite et évacue pour un temps l'angoisse de l'inconnu et l'angoisse de mort, a plusieurs conséquences, notamment:

- Le désinvestissement de l'énigme du Sphinx - le temps linéaire et la finitude de la vie humaine - au profit du surinvestissement de l'agir immédiat, vécu comme intemporel.

- L'accroissement du déni du principe de réalité et, en tout premier lieu, de la réalité de la mort de l'individu.

- Corrélativement, les mythes de transformation et de renaissance privilégient la qualité technologique de la mutation, au détriment de la qualité relationnelle.

Cependant, ce nouvel équilibre défensif incluant le virtuel n'est guère efficace. L'enfant qui se réfugie dans les jeux d'exploits ou de combats de sa console de jeux ne peut plus en ressortir, sous peine de retrouver son angoisse, d'autant plus primaire qu'il rentre dans une réalité toujours non digérée. Le psychanalyste devra faire patiemment son chemin, à partir de ces fictions, jusqu'aux angoisses existentielles classiquement proposées par la dialectique fantasme/réalité de l'être humain.

\section{Angle dynamique et économique}

Ce désinvestissement de la vie psychique interne s'accompagne d'une pathologie du refoulement et, par conséquent, d'une désorganisation du développement en deux temps décrit par Freud dans son modèle de la névrose infantile. L'après-coup (Nachträglichkeit) tel qu'il l'entendait, ne se constitue plus de la même façon, puisque les modes infantiles de la sexualité demeurent manifestes de façon continue entre l'âge de l'Edipe et la puberté.

On observe notamment une excitabilité sans limites de la génitalité infantile, caractérisée par un mimétisme de la sexualité adulte, expression directe du déni de la différence des générations. Les enfants ne vivent plus leur enfance, et l'on peut se demander si leur apparente hyper maturité n'est pas souvent une pseudo maturité. En effet, les affects dépressifs sont évacués dans l'hyperactivité - jusqu'au burn out ou au break down suicidaire.

\section{Angle topique et structural}

Les éléments œdipiens, présents et actifs à partir de la seconde moitié de la première année de vie, ne s'organisent pas en complexe d'Edipe, pas plus que la problématique de la castration ne se structure en véritable complexe de castration. La 
relation d'intimité, pierre de touche d'une structure psychique véritablement génitale, ne pourra pas prendre place dans la deuxième partie de l'adolescence et dans les débuts de la vie sexuelle adulte. Elle sera remplacée par la pérennité des valeurs phalliques et groupales qu'est la recherche de l'exploit, dans une mentalité voyeuriste/ exhibitionniste.

Le mode de fonctionnement de ces jeunes patients présente des analogies, mais aussi des différences, avec celui que l'École française de Psychosomatique a désigné comme «pensée opératoire» (Marty, 1991).

L'une des différences réside dans le « rattrapage spéculaire» qu’ils parviennent parfois à effectuer, lorsqu'ils trouvent un «miroir psychique» suffisamment proche et disponible dans leur entourage ou chez l'analyste. En cela, leur mode de pensée m'évoque celui des autistes, et également, dans une certaine mesure, celui de la «mentalité de groupe » décrite par Bion.

Surtout, il me fait penser au fonctionnement en logique binaire, que j'ai décrit plus haut à propos de l'intelligence artificielle et du virtuel. Et c'est en partant de ce paramètre que je voudrais brièvement indiquer quelles sont les difficultés auxquelles les enfants d'aujourd'hui confrontent leurs psychanalystes.

\section{Conséquences pour la clinique psychanalytique}

Face à cette nouvelle distribution des éléments constitutifs du fonctionnement psychique, au relais endémique du niveau primaire des défenses du Moi (clivage, déni, idéalisation, projection identificatoire ${ }^{5}$ ) que constitue le virtuel dans la culture actuelle, alors que le niveau secondaire de celles-ci (défenses liées au refoulement secondaire (Freud, 1915-1917)) fond comme neige au soleil, le psychanalyste va rencontrer deux ordres de difficultés :

1. Difficultés d'ordre contretransférentiel;

2. Difficultés d'ordre technique.

\section{Difficultés d'ordre contretransférentiel}

Notre analyse personnelle nous a donné la capacité de prendre conscience des éléments de notre propre Infantile lorsqu'il entre en résonance avec celui de notre analysant, quel que soit l'âge de ce dernier et, a fortiori, avec l'Infantile de nos patients enfants. Nous sommes donc en mesure d'aller et venir au travers de la barrière analysée de notre propre refoulement, voire de nos propres clivages. Néanmoins, notre mouvement intérieur fonctionne principalement sur un modèle névrotique : celui de notre névrose de contre-transfert. C'est, notamment, ce modèle qui est responsable de la transformation de nos théories sexuelles infantiles dans ce qui est devenu notre théorie analytique implicite.

Certes, l'entraînement que nous avons acquis dans l'utilisation de notre projection identificatoire - en principe normale - va prendre le relais de ce fonctionnement névrotique. Mais il nous entraînera alors vers d'inévitables et indispensables taches aveugles dans notre contre-transfert (Guignard, 2002b). Ces dernières nous conduiront à penser, voire à agir verbalement, en projection identificatoire avec celui 
des objets internes du patient qui se trouve être le plus actif - et pas nécessairement le plus manifeste - sur la scène analytique du moment ${ }^{6}$.

Cette tension entre notre propre Infantile analysé et celui de notre patient est indispensable au maintien et à l'analyse du lien analytique. Elle sera d'autant plus difficile à vivre que nous serons confrontés à des mécanismes de défense archaïques, plutôt qu'au refoulement secondaire. Ceci constitue l'une des difficultés majeures de l'exercice de la psychanalyse avec les enfants, et rend compte de la résistance considérable de notre communauté psychanalytique à cette pratique.

L’une de nos issues défensives les plus fréquentes consiste à utiliser des «interprétations-bouchons » (Guignard, 2002c), pour lesquelles nous faisons appel notamment:

- à l'évocation de l'histoire personnelle de l'analysant et à la représentation aléatoire que nous nous faisons des personnes réelles de cette histoire;

- à nos connaissances théoriques et, par conséquent, à notre propre théorie analytique implicite.

La sortie d'une tache aveugle se produit, chez l'analyste, dans un mouvement de protestation identitaire par lequel il se dégage de sa projection identificatoire aux objets internes du patient. Cette protestation identitaire est tout particulièrement sollicitée lorsque le patient présente une utilisation pathologique des mécanismes primaires d'organisation de son Moi, sous forme d'une régression à un état passager de confusion durant la séance.

En d'autres termes, la désintrication pulsionnelle du patient suscite chez l'analyste un mouvement de déni de l'angoisse de mort, qui constitue le primum movens du développement psychique.

Confronté à ces configurations, particulièrement présentes et actives chez l'enfant et chez l'adolescent, le psychanalyste devra effectuer un important travail autoanalytique pour réinstaller sa capacité de rêverie, activité préconsciente dont l'illustration princeps demeure pour moi le «Père, ne vois-tu pas...» du rêve rapporté par Freud (1900). Je rappelle que cette capacité de rêverie est l'expression d'un psychisme adulte, au Complexe d'Edipe organisé.

Le but premier de l'analyse devient dès lors de favoriser l'activité de symbolisation chez le patient, introduisant par là le tiers indispensable au développement névrotico-normal.

Pourtant, il se pourrait que nos capacités d'identification aux enfants des générations présentes et à venir soient battues en brèche par les profondes modifications de leur vision du monde, dans laquelle la capacité de symboliser va peut-être tenir un rôle moins important que par le passé, face à l'importance que prend pour eux le monde virtuel. En d'autres termes, notre but de psychanalystes - aider à installer et à organiser une vie psychique interne, c'est-à-dire, ce qu'il y a de plus intime et de plus personnel chez un individu - sera-t-il toujours intéressant aux yeux des générations à venir, qui sont prises dans une mondialisation de schémas virtuels tout faits d'avance? 


\section{Difficultés d'ordre technique}

Dans le monde où vivent les enfants et les adolescents d'aujourd'hui, et où prévalent:

- l'expression du virtuel sur l'expression du fantasmatique;

- l'utilisation d'un système binaire d'équation symbolique - ou, si l'on veut faire référence à Peirce, d'indices et d'icônes - sur l'utilisation d'une symbolisation à trois termes,

... quels outils techniques le psychanalyste va-t-il pouvoir utiliser afin d'établir des liens entre ces deux modes de fonctionnement, si différents l'un de l'autre?

Je pense que la mondialisation du système de logique binaire utilisé dans l'imagerie virtuelle est l'occasion, pour les psychanalystes, de considérer sous un angle nouveau les recherches qui ont été effectuées dans le monde de l'autisme. Je pense notamment aux travaux de D. Meltzer (1975), de F. Tustin (1972, 1981, 1986) et de G. Haag (1985). Il serait temps que la psychanalyse accorde toute son attention à cette autre forme d'intelligence et de Weltanschauung, sans abandonner, pour autant, ses paramètres psychanalytiques et, en particulier, la théorie de la pensée élaborée par W. R. Bion.

Il ne suffit plus, aujourd'hui, de repérer des «parties psychotiques» et des «barrières autistiques » chez des patients enfants et adolescents que nous continuerions à considérer à l'aune d'un développement névrotique classique. Nous devrions nous attacher à comprendre de façon suffisamment approfondie ce qui, dans le monde de logique binaire du virtuel, fonctionne comme un attracteur si puissant sur les jeunes patients, qu'il leur permet d'éviter le travail psychique de symbolisation lié à l'internalisation de l'objet et, partant, à l'élaboration de la perte de celui-ci.

Dans cette perspective, et si nous croyons toujours à la force d'intrication pulsionnelle que constitue la talking cure, il devient tout particulièrement important de nous pencher sur les pathologies du sadisme primaire, expression première de l'intrication des pulsions de vie et de mort, comme Mélanie Klein l'a si bien décrit (Klein, 1927) en relation avec les tendances criminelles, qui nous posent également de plus en plus de problèmes de société.

Résultant de cette première intrication, l'organisation du sadisme oral et anal est le lieu par excellence où l'influence de l'organisation pulsionnelle du monde adulte va exercer son action sur l'organisation psychique de l'enfant. C'est aussi, selon moi, le lieu où le monde du virtuel va proposer son échappatoire omnipotente et manichéenne à nos jeunes patients.

Par ailleurs, en l'absence d'une véritable période de latence, le psychanalyste des enfants d'aujourd'hui doit apprendre à écouter les micromouvements d'après-coup qui se produisent dans le champ analytique. En effet, tout se passe comme si les enfants devaient trouver une nouvelle façon de transformer leur excitation sexuelle, sans avoir recours de façon majeure au refoulement. Le risque réside évidemment dans l'utilisation préférentielle des mécanismes de défense primaires. 


\section{Conclusion}

La psychanalyse de l'enfant est une pratique de pointe sur le plan thérapeutique, et elle constitue, de ce fait, le champ le plus avancé de la recherche en psychanalyse. Nous serons donc les premiers à découvrir les structures psychiques que les enfants d'aujourd'hui et les adultes de demain organiseront en lieu et place de la névrose infantile que nous avons connue jusqu'ici.

C'est une occasion à ne manquer sous aucun prétexte. Pour cela, nous devons affiner nos moyens d'observation et, notamment, utiliser pleinement les éléments de la psychanalyse installés par les continuateurs de l'œuvre freudienne, notamment Klein et Bion. Par exemple, je crois que les concepts tels que la position dépressive centrale de Klein et la relation contenant/contenu de Bion peuvent encore nous servir de repères fondamentaux dans notre pratique quotidienne, car l'effondrement menace plus que jamais les générations à venir, notamment parce que la société d'aujourd'hui et de demain ne brille pas par un excès d'activité psychique contenante.

Cependant, si nous voulons maintenir notre écoute à son meilleur niveau, «sans mémoire ni désir » (Bion), nous aurons à suspendre nos perspectives théoriques classiques, tout au moins celle du modèle de la névrose comme référence de la normalité et résister à la tentation de fermer le champ de notre recherche en y substituant un autre modèle déjà connu pour apaiser notre angoisse.

Pour autant, nous ne nous referons pas, et nous ne pourrons nous empêcher d'investir ce monde psychique interne qui constitue notre matériau spécifique. Dans cette perspective, nous devrons nous intéresser suffisamment longtemps à la scène du virtuel que nous propose l'enfant, pour que les «objets inanimés» qui la constituent acquièrent « une âme ${ }^{7}$ ». C'est à ce prix que ceux-ci quitteront leur place d'objets préfabriqués pour devenir des représentants des émotions, plutôt que de demeurer des évacuations sous forme d'actions. Mais nous devons être plus prudents que jamais: dans ce contexte nouveau, si nous établissons trop vite des liens entre les intérêts de l'enfant et la situation de transfert, le champ analytique pourrait bien se rétrécir comme une peau de chagrin, et le risque d'une réaction thérapeutique négative surgir inéluctablement. L'interprétation prématurée du transfert agit, dans ce mode nouveau de fonctionnement psychique, comme une séduction sexuelle et inhibe très rapidement le mécanisme de base du développement psychique que constitue le déplacement.

Florence Guignard Square d'Orléans

80 rue Taitbout 75009 PARIS courriel : flogui2@club-internet.fr

\section{Notes}

1. La presse belge vient de relater le cas d'une internaute qui a intenté un procès à un autre internaute, lui reprochant d'avoir violé son avatar. On imagine le champ juridique que cela ouvrirait si un tribunal estimait que l'affaire devait être jugée et si l'internaute était condamné. 
2. «Les Trente Glorieuses » : Expression de l'économiste français Jean Fourastié pour désigner les trente années de prospérité en Occident, qui s'étendent de 1945 à 1974. Ces trente années ont notamment vu: les premières bombes à hydrogène (Hiroshima et Nagasaki 1945), la guerre d'Algérie (19541962), la guerre franco-indochinoise (1945-1954) suivie de la guerre américano-vietnamienne (1959-1975), et l'assassinat de John Fitzgerald Kennedy (1963). 1968 fut notamment l'année du «Printemps de Prague», de l'assassinat de Martin Luther King, de la découverte du génome humain, du mouvement de mai 68 en France et du mouvement hippie né aux États-Unis, avec la création de Hair, suivi de Woodstock en 1969. Enfin, 1974 vit tomber les deux dernières dictatures d'Europe de l'Ouest: au Portugal, (la « révolution des œillets ») et en Grèce (le régime des colonels).

3. Voir notamment Sherry Turckle, 1997, Life on the Screen. Identity in the Age of Internet, ainsi que plusieurs ouvrages récents de Serge Tisseron, de même que l'ouvrage collectif de S. Tisseron, S. Missonier et M. Stora, 2006, L'enfant au risque du virtuel, Dunod, Paris. Voir également ma conférence (non publiée) donnée au CAPSA au Congrès de l'API à Berlin en 2007.

4, Voir S. Korff Sausse, 2006, L'Enfant Roi, PUF, Paris.

5. J'utilise cette traduction de "projective identification », plus correcte, à mon sens, que le terme habituel d' «identification projective».

6. Le concept de «tache aveugle dans le contre-transfert» se rapproche du concept de «contre-identification projective » de L. Grinberg, sans pourtant s'y superposer entièrement.

7. Lamartine, A. de, 1826, Milly ou la terre natale : «Objets inanimés, avez-vous donc une âme. Qui s'attache à notre âme et la force d'aimer?».

\section{Références}

BION, W. R., 1959/1965, Recherches sur les petits groupes, PUF, Paris.

BLEGER, J., 1981, Symbiose et ambiguité, PUF, Coll. Le fil rouge, Paris.

CASTORIADIS, C., 1996, La montée de l'insignifiance, Les carrefours du labyrinthe 4, Seuil, Paris.

FERENCZI, S., 1932/1982, Confusion de langues entre les adultes et les enfants, Le langage de la tendresse et de la passion, Psychanalyse IV, Payot, Paris, 125-135.

FREUD, S., 1900/1967, L'interprétation des rêves, PUF, Paris.

FREUD, S., 1905/1962, Trois essais sur la théorie de la sexualité, Gallimard, Paris.

FREUD, S., 1915/1985, Vue d'ensemble des névroses de transfert. Un essai métapsychologique, Édition bilingue d'un manuscrit retrouvé, Gallimard, Paris.

FREUD, S., 1925/1992, La négation, O.C.F. XVII, PUF, Paris.

GUIGNARD, F., 1990, Devenir adulte? Apprendre l'incertitude, in Alléon A. M., Lebovici S. et al., eds., Devenir adulte?, PUF, Paris, 123-141.

GUIGNARD, F., 1996, Au vif de l'Infantile. Réflexions sur la situation analytique, Delachaux et Niestlé, Coll. Champs psychanalytiques, Lausanne.

GUIGNARD, F., 2002a, Le sadisme au service de la vie. De la morsure au clivage, de l'expulsion au déni, de la rétention à la transformation psychique, Revue française de psychanalyse, 4/02.

GUIGNARD, F., 2002b, Les concepts métapsychologiques de troisième type, in BARANÈS,

J.-J., SACCO, F., et al., eds., Inventer en psychanalyse. Construire et interpréter, Dunod, Paris, 99-113.

GUIGNARD, F., 2002c, Apories de la transformation dans l'activité psychique du psychanalyste en exercice: taches aveugles et interprétations-bouchons, Revue française de psychanalyse, LXVI, $\mathrm{n}^{\circ}$ spécial Congrès, P.U.F, Paris.

GUIGNARD, F., 2006, La pensée de Jean Laplanche. Convergences et apories, Psychiatrie française, 3, 90 109.

HAAG, G., 1985, Psychothérapie d'un enfant autiste, Approche psychanalytique de l'autisme infantile, Lieux de l'Enfance, 3, Privat, Toulouse, 65-78.

KLEIN, M., 1927/1967, Les tendances criminelles chez les enfants normaux, Essais de psychanalyse, Payot, Paris, 211-228.

KORFF-SAUSSE, S., 2006, L'Enfant Roi, PUF, Paris.

MARTY, P., 1991, Mentalisation et psychosomatique, Les empêcheurs de penser en rond, Paris. 
MELTZER, D., et al., 1975, Explorations in Autism, Clunie Press, Perthshire.

MELTZER, D., 1977, Les structures sexuelles de la vie psychique, Payot, Paris, 1973.

ORTOLI, S., PHARABOD, J.-P, 1984, Le Cantique des Quantiques, La Découverte, Paris.

SALOMONSSON, B., 2006, The impact of words on children with ADHD and DAMP, International Journal of Psychoanalysis, 87, part 4. Trad. F. Guignard in L'Année Psychanalytique Internationale 2007 p. 99-118, Paris, In Press, 2007.

TURKLE, S., 2009, Simulation and Its Discontents, in Clancey, W. J., Helmreich, S., Loukissas, Y. A., Myers, N., Maeda, J., eds., Essays, The MIT Press, Boston.

TUSTIN, F., 1972, Autism and Childhood Psychosis, Hogarth Press, London;

TUSTIN, F., 1981, Autistic States in Children, Routledge, London.

TUSTIN, F., 1986, Autistic Barriers in Neurotic Patients, Hogarth Press, London.

WINNICOTT, D. W., 1958/1969, De la pédiatrie à la psychanalyse, Payot, Paris. 